\title{
Understanding Reversals of a Rattleback
}

Stefan Rauch-Wojciechowski and Maria Przybylska

The self-archived postprint version of this journal article is available at Linköping University Institutional Repository (DiVA):

http:/ / urn.kb.se/ resolve?urn=urn:nbn:se:liu:diva-140526

N.B.: When citing this work, cite the original publication.

Rauch-Wojciechowski, S., Przybylska, M., (2017), Understanding Reversals of a Rattleback,

Regulârnaâ i haoticeskaâ dinamika, 22(4), 368-385. https:// doi.org/ 10.1134/ S1560354717040037

Original publication available at:

https:/ / doi.org/ 10.1134/ S1560354717040037

Copyright: MAIK Nauka/ Interperiodica http:/ / www.maik.rssi.ru/ ru/journals/ $\Rightarrow$ Tweet 


\title{
Understanding reversals of a rattleback
}

\author{
Stefan Rauch-Wojciechowski \\ Department of Mathematics, Linköping University, 58183 Linköping, Sweden \\ e-mail: strauc@mai.liu.se
}

\author{
Maria Przybylska \\ Institute of Physics, University of Zielona Góra, Licealna 9, PL-65-417 Zielona Góra, Poland \\ e-mail: M.Przybylska@if.uz.zgora.pl
}

May 4, 2017

\begin{abstract}
A counterintuitive unidirectional (say counterclockwise) motion of a toy rattleback takes place when it is started by tapping it at a long side or by spinning it slowly in the clockwise sense of rotation. We study motion of a toy rattleback having ellipsoidal shaped bottom by using frictionless Newton equations for motion of a rigid body rolling without sliding in a plane. We simulate these equations for tapping and spinning initial conditions to see the contact trajectory, the force arm and the reaction force responsible for torque turning the rattleback in the counterclockwise sense of rotation. Long time behaviour of such rattleback is however quasiperiodic and a rattleback starting with small transversal oscillations turns in the clockwise direction.
\end{abstract}

Mathematics Subject Classification: 37J60; 37J25; 70G45

Keywords: rattleback, rigid body dynamics, nonholonomic mechanics, numerical solutions

\section{Introduction}

A toy rattleback is a body having shape of an elongated boat that displays the rattleback effect that when it is spun about the vertical axis $\hat{\mathbf{z}}$ in the wrong (say clockwise) direction then it slows down, starts to rattle in longitudinal direction and from this motion acquires the preferred (counterclockwise) direction of rotation. This behavior is counterintuitive, raises curiosity and seems to be difficult to explain to a laymen using basic laws of Newtonian mechanics. The rattleback effect is more transparent when the rattleback is started by tapping a long edge that makes it to oscillate. Then the oscillation amplitude gradually decreases as the rattleback acquires counterclockwise rotation. Due to presence of friction and, therefore, dissipation of energy this rotation slows down and after some time stops. Friction force is always present in any experimental demonstration but it does not seem to be essential for understanding reversals of the rattleback [3].

An intuitive understanding of the rattleback effect requires reconciliation of the observed behavior with our everyday experience about conservation of angular momentum. A change of angular momentum requires a source of torque, which is not directly visible in the rattleback case as it is the reaction force of the supporting surface that gives rise to the torque.

The purpose of this note is to discuss within vectorial mechanics direction of the reaction force, the force arm and the torque in the body to see how this torque works in the preferred direction and reverses sense of rotation. This understanding is based on numerical simulations of a realistic 
toy rattleback starting with oscillatory initial conditions (IC) or with a slow clockwise rotation. In a frictionless model rattlebacks energy is conserved and it appears that for low energy initial conditions also back reversals take place when a rattleback is started with transversal initial oscillations. Long time solutions appear to be quasiperiodic in agreement with integrable Markeev equations [16].

Analysis of rattleback behavior is usually associated with question of linear stability of exact solutions representing two vertical spinning solutions in either direction. This has been for the first time discussed 1896 by G.T.Walker [19] who considered a paraboloidal shaped rigid body rolling without sliding of the contact point. He analysed equations for the contact point variables and the vertical spin variable that were linearized about two vertical spinning solutions and he formulated conditions of stability of these solutions. In [3] this analysis has been extended to determine dependence of stability, for vertical spinning solutions, on the moments of inertia and on curvatures of rattlebacks bottom surface. H. Bondi also asserted that slipping and friction is unimportant in understanding dynamics of a rattleback, see also [9]. A rigorous linear stability analysis of vertical spinning solutions has been addressed in $[1,13]$ where the condition for relation between spinning velocity, main curvatures of a convex body (ellipsoid), the moments of inertia and the chirality parameter (angle) have been formulated. In [16] there has been derived a set of 3 nonlinear equations for small perturbations about stable equilibrium to study coupling between the modes of longitudinal and transversal oscillations. Remarkably, these equations are integrable and the value of energy integral depends on the value of spinning velocity about vertical axis. This idea has been developed to include friction by Takano who attempted to estimate a number of admissible reversals [18].

Most of this analysis concerned linear stability of the spinning solutions, the stability of oscillatory components in the rattleback motion and interaction between these energy modes to explain why and when there is unidirectional preference of rattlebacks motion. The torque and the reaction force responsible for rattleback reversals has been discussed only in a few papers e.g. [10, 11] and there is seemingly no vectorial discussion of how they work and come up during reversals.

The linearized and/or simplified equations of motion cannot predict all phenomena in ratteback motion and several papers analyzed dynamics of a rattleback using numerical simulations of full equations $[15,5,9]$. Last decade, due to work of Borisov, Mamaev and their collaborators, it became apparent that global dynamics of a rattleback is very rich. Apart from the well-known stable and unstable stationary equilibrium rotations about the vertical principal inertia axis there may exist limit cycle solutions and even strange attractors $[9,12,4]$. It appears that reversal phenomena in dynamics of nonholonomic rigid bodies are quite common and have been discovered in motion of Chaplygin ball [6] and Suslov top [2]. In a more general framework of nonholonomic systems on a Lie algebra change of stability of stationary motions and reversion of direction of motion has been discussed in [14]. Further references about rattleback dynamics may be found in review papers $[9,7]$.

In Sec. 2 we formulate Newton equations for a rattleback rolling without sliding and discuss direction of contact trajectory, direction of reaction force and direction of torque in case of longitudinal and transversal oscillations. This provides an elementary way of understanding rattleback reversals, which is accessible for a laymen. In Sec. 3 we specify physical parameters: shape, size, distribution of mass and moments of inertia of a toy rattleback that is simulated and discussed in Sec. 4. Also conditions of linear stability of this rattleback are given.

For numerical simulations in Sec. 4 we use rattleback equations with respect to (w.r.t.) a bodyfixed reference frame [9]. As being globally defined they are better suited for numerical calculation of solutions. The general picture is that for low energy initial conditions motion of this rattleback is quasiperiodic as energy is conserved and for low energies neither of 2 spinning solutions is stable. We discuss mainly motion starting with slow clockwise rotation as it shows how the rattleback acquires counterclockwise torque and changes the sense of rotation. From this solution it is also possible to read off what happens for longitudinal and for transversal oscillatory initial conditions. Study of initial oscillatory solutions generated by tapping a rattleback on the side at different angles $\phi(0)$ w.r.t. long axis $\hat{\xi}$ shows that for prevalent region of angles the rattleback acquires counterclockwise torque [15] but clockwise torque is also possible when transversal oscillations dominate. Our simulations show that there are 4 directions of oscillatory (tapping) initial conditions when the rattleback only oscillates and has no preferred direction of rotation. 


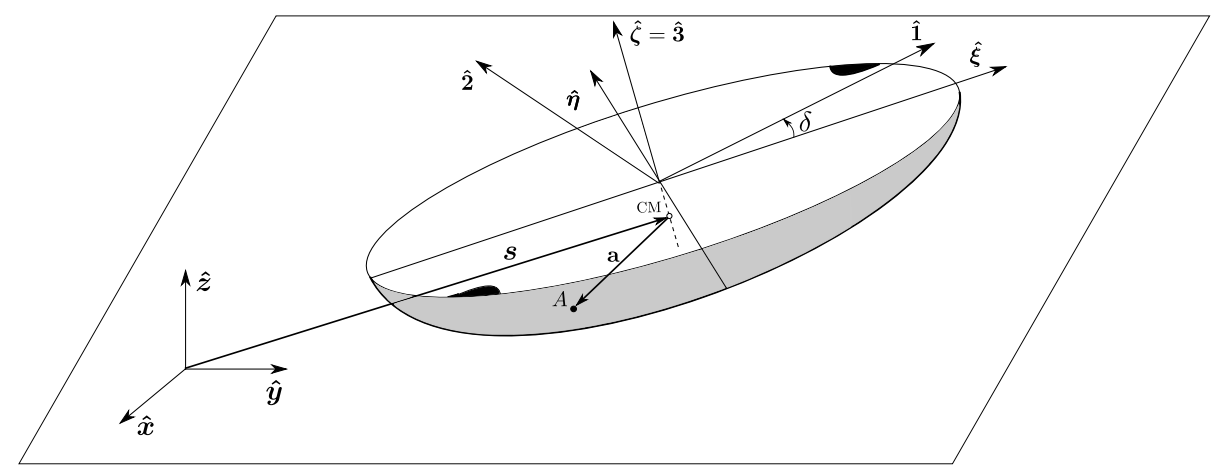

Figure 1: Geometry of the rattleback. In this picture $\hat{\mathbf{1}}, \hat{\mathbf{2}}, \hat{\mathbf{3}}$ denote the principal axes of inertia turned counterclockwise by a small angle $\delta$ with respect to axes $\hat{\xi}, \hat{\eta}$ directed along the geometrical axes of an tri-axial ellipsoid.

\section{Intuitive understanding of rattleback reversals}

We consider here a rattleback having shape of an halv-ellipsoid with skew-symmetric distribution of mass such that axes of the main moments of inertia are rotated with respect to the main geometrical axes. To describe motion of the rattleback we use three reference frames. A fixed inertial reference frame $(\hat{\mathbf{x}}, \hat{\mathbf{y}}, \hat{\mathbf{z}})$ has basis vectors $\hat{\mathbf{x}}, \hat{\mathbf{y}}$ in the plane of support and vertical axis $\hat{\mathbf{z}}$; hat means here a unit vector. We choose two right-handed reference frames $(\hat{\boldsymbol{\xi}}, \hat{\boldsymbol{\eta}}, \hat{\zeta})$ and $(\hat{\mathbf{1}}, \hat{\mathbf{2}}, \hat{\mathbf{3}})$ fixed in the body, see Fig. 1. The axis $(\hat{\xi}, \hat{\eta}, \hat{\zeta})$ are directed along the main geometrical semi-axes $b_{1}>b_{2}>b_{3}$ of the ellipsoid so that $\hat{\zeta}$ is pointing upwards when the rattleback is standing still and $\hat{\zeta}$ is pointing along the long axis $b_{1}$. The axes $(\hat{\mathbf{1}}, \hat{\mathbf{2}}, \hat{\mathbf{3}})$ point along the directions of the main moments of inertia. We assume that the distribution of mass in the rattleback is such that the center of mass (CM) is placed on the halvaxis $b_{3}$ and it is shifted down by $d<b_{3}$ with respect to the flat top surface of the halv-ellipsoid. The main axis of inertia $\hat{\mathbf{3}}$ coincides with the axis $\hat{\zeta}$ and $\hat{\mathbf{1}}, \hat{\mathbf{2}}$ stay in the same flat top plane of the rattleback being rotated counterclockwise by a small angle $\delta>0$ with respect to the axis $\hat{\xi}$, $\hat{\eta}$. So that $\hat{\mathbf{1}}=\hat{\boldsymbol{\xi}} \cos \delta+\hat{\eta} \sin \delta$, $\hat{\mathbf{2}}=-\hat{\boldsymbol{\xi}} \sin \delta+\hat{\eta} \cos \delta$ and $\hat{\mathbf{3}}=\hat{\boldsymbol{\zeta}}$.

We consider motion described by the Newton equations for a rolling rattleback without taking into account the sliding friction which is understood as not being essential factor for explaining reversals of a rattleback. In such model there are two forces acting on the rattleback: the gravitational force $-m g \hat{\mathbf{z}}$ and the force $\mathbf{F}_{\mathrm{R}}$ of reaction of the supporting surface, which at each moment of time keeps the rattleback rolling without sliding.

The equations of motion are

$$
m \ddot{\mathbf{s}}=\mathbf{F}_{\mathrm{R}}-m g \hat{\mathbf{z}}, \quad \dot{\mathbf{L}}=\mathbf{a} \times \mathbf{F}_{\mathrm{R}}, \quad \dot{\hat{\mathbf{1}}}=\boldsymbol{\omega} \times \hat{\mathbf{1}}, \quad \dot{\hat{\mathbf{2}}}=\boldsymbol{\omega} \times \hat{\mathbf{2}}, \quad \dot{\hat{\mathbf{3}}}=\boldsymbol{\omega} \times \hat{\mathbf{3}},
$$

where $\mathbf{s}$ denotes position of the center of mass (CM) w.r.t. the inertial reference frame $(\hat{\mathbf{x}}, \hat{\mathbf{y}}, \hat{\mathbf{z}}), \mathbf{a}$ is a vector from $\mathrm{CM}$ to the instantaneous point of support A (see Fig. 1), $\mathbf{L}=\mathbb{I} \boldsymbol{\omega}$ is the angular momentum and $\mathbb{I}$ is the tensor of inertia.

The pure rolling condition means that the sliding velocity vanishes identically in time $\mathbf{v}_{A}(t)=$ $\dot{\mathbf{s}}(t)+\boldsymbol{\omega}(t) \times \mathbf{a}(t)=\mathbf{0}$. Then $\dot{\mathbf{s}}=-\boldsymbol{\omega} \times \mathbf{a}$ and the reaction force $\mathbf{F}_{\mathbf{R}}=m \ddot{\mathbf{s}}+m g \hat{\mathbf{z}}=m(\mathbf{a} \times \boldsymbol{\omega})^{\dot{1}}+m g \hat{\mathbf{z}}$ is dynamically determined so that

$$
\dot{\mathbf{L}}=\mathbf{a} \times \mathbf{F}_{\mathrm{R}}=m \mathbf{a} \times\left[(\mathbf{a} \times \boldsymbol{\omega})^{\dot{0}}+g \hat{\mathbf{z}}\right], \quad \dot{\hat{\mathbf{3}}}=\boldsymbol{\omega} \times \hat{\mathbf{3}}=\mathbb{I}^{-1} \mathbf{L} \times \hat{\mathbf{3}} .
$$

The energy of the system is a sum of kinetic energy $E_{\mathrm{CM}}$ of translational motion of the mass center, rotational energy $E_{\text {rot }}$ about $\mathrm{CM}$ and of potential energy $E_{\text {pot }}$ of $\mathrm{CM}$

$$
E=E_{\mathrm{CM}}+E_{\mathrm{rot}}+E_{\mathrm{pot}}=\frac{1}{2} m \dot{\mathbf{s}}^{2}+\frac{1}{2} \omega \llbracket \boldsymbol{\omega}+m g \mathbf{s} \mathbf{z} .
$$


The energy is conserved as

$$
\begin{aligned}
\dot{E} & =\frac{\mathrm{d}}{\mathrm{d} t}\left(\frac{1}{2} m \dot{\mathbf{s}}^{2}+\frac{1}{2} \boldsymbol{\omega} \mathbf{L}+m g \mathbf{s} \hat{\mathbf{z}}\right)=\dot{\mathbf{s}}\left(\mathbf{F}_{\mathrm{R}}-m g \hat{\mathbf{z}}\right)+\boldsymbol{\omega}\left[\mathbf{a} \times \mathbf{F}_{\mathrm{R}}\right]+m g \dot{\mathbf{s}} \hat{\mathbf{z}}=\left(\mathbf{v}_{A}-\boldsymbol{\omega} \times \mathbf{a}\right) \mathbf{F}_{\mathrm{R}} \\
& -m g \dot{\mathbf{s}} \hat{\mathbf{z}}+\boldsymbol{\omega}\left[\mathbf{a} \times \mathbf{F}_{\mathrm{R}}\right]+m g \dot{\mathbf{s}} \hat{\mathbf{z}}=\mathbf{v}_{A} \mathbf{F}_{\mathrm{R}}=0 .
\end{aligned}
$$

In this calculation $m g \dot{\mathbf{s}} \hat{\mathbf{z}}$ is the rate of energy transfer into potential energy $E_{\text {pot }}$ and $\omega\left[\mathbf{a} \times \mathbf{F}_{R}\right]$ is the work performed in unit time by the torque $\mathbf{a} \times \mathbf{F}_{\mathrm{R}}$ that transfers energy between $E_{\mathrm{CM}}$ and $E_{\text {rot }}$.

In order to understand the rattleback effect of unidirectional (counterclockwise) preference of rotation one needs to see the torque responsible for turning longitudinal, rolling oscillations into the counterclockwise rotational motion with decreasing amplitude of oscillations and increasing spin velocity as the total energy in this model is conserved.
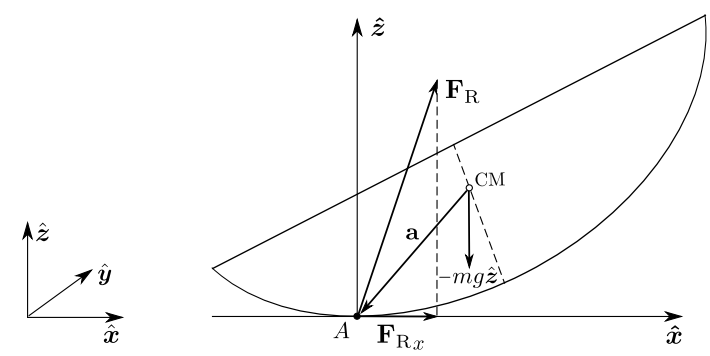

Figure 2: Oscillation of a symmetric rattleback.

Longitudinal oscillations of a symmetric rattleback Take first a rattleback with symmetric distribution of mass (with respect to the vertical plane going through the long axis of the ellipsoid) set into longitudinal oscillations, which is rocking without acquiring rotational motion in either direction. This means that we consider rocking as a 2-dimensional planar body. This situation is illustrated in Fig. 2 where a rattleback standing on the left side is first moving leftwards with decelerating speed $\dot{\mathbf{s}}$ of center of mass (CM) until it stops. From this position CM is accelerated in the positive $\hat{\mathbf{x}}$-direction and acquires maximal speed when $\mathrm{CM}$ is passing about the contact point $\mathrm{A}$. Then the two first phases of this motion are repeated during motion in the opposite direction. During phase 1 and 2, when the rattleback is standing on the left side as in Fig. 2, the reaction force $F_{R}$ is pointing upwards within the angle between the vertical axis $\hat{\mathbf{z}}$ and the line determined by the vector $\mathbf{a}$. To understand this see from the Newton equation (2.1) for CM that the vertical component of the reaction force $\mathbf{F}_{\mathbf{R}} \hat{\mathbf{z}}=m g+m \ddot{\mathbf{s}} \hat{\mathbf{z}}$ compensates the weight $m g$ and is modified by the term $m \ddot{\mathbf{s}} \hat{\mathbf{z}}$, which arises due to positive (or negative) acceleration of $\mathrm{CM}$ in the vertical direction. The horizontal component of the reaction force $F_{\mathrm{R} x}=$ $\mathbf{F}_{\mathrm{R}} \hat{\mathbf{x}}=m \ddot{\mathbf{s}} \hat{\mathbf{x}}$ must act in the right direction to decelerate $\mathrm{CM}$ in phase 1 and accelerate $\mathrm{CM}$ in the positive direction of $x$ during phase 2 . This means that in phase 1 and 2 the component $F_{\mathrm{R} x}$ has to point to the right in Fig. 2. But the value $F_{R x}$ can not be too large since the torque $\mathbf{T}=\mathbf{a} \times \mathbf{F}_{\mathrm{R}}$ has to point behind the plane of Fig. 2 to decelerate rotation of rattleback in phase 1 and accelerate rattleback in the clockwise direction during phase 2 . Thus the value of $F_{\mathrm{R} x}$ is bounded by the condition of having $\mathbf{F}_{\mathrm{R}}$ in the angle between $\hat{\mathbf{z}}$ and the line along vector $\mathbf{a}$.

In the next two phases 3 and 4 of oscillation the vectors $\mathbf{a}$ and $\mathbf{F}_{\mathrm{R}}$ are mirror images of the situation in Fig. 2.

Source of torque turning rattleback counterclockwise during longitudinal oscillations. When to a reflectionally symmetric, boat shaped rattleback two extra weights are added asymmetrically as in Fig. 3a the rattleback acquires "chirality" and tends to turn in the counterclockwise direction.

A symmetric rattleback admits a longitudinal oscillating solution along a straight line of contact with the bottom of the long symmetry line of the ellipsoid. When extra (black) weights are added, then during the left side oscillation in $\hat{\xi}$ direction the contact line of the bottom of ellipsoid deflects on the body surface along a dotted line indicated in Fig. 3a by vector $\mathbf{a}$. 
In order to have a torque accelerating rattleback in the counter clockwise direction the $(\hat{\xi}, \hat{\boldsymbol{\eta}})$-plane component of force $\mathbf{F}_{\mathrm{R} \|}=\mathbf{F}_{\mathrm{R}} \hat{\boldsymbol{\xi}}+\mathbf{F}_{\mathrm{R}} \hat{\boldsymbol{\eta}}$ has to be in Fig. 3a directed below the dashed line extending vector a. It is visible in the simulation in Fig. 4a where the left longer arm L of the cross-shaped trajectory of $\mathbf{a}_{\xi \eta}(t)=(\mathbf{a} \hat{\xi}) \hat{\xi}+(\mathbf{a} \hat{\eta}) \hat{\eta}$ is pointing above the long axis of the ellipse. The corresponding $\operatorname{arm} \mathrm{R}_{\mathrm{L}}$ of the reaction force trajectory $\mathbf{F}_{\mathrm{R} \|}(t)$ describes force pointing downward according to Fig. $4 \mathrm{~b}$. This provides a positive vertical component of torque $\mathbf{T} \hat{\zeta}$ and also $\mathbf{T} \hat{\mathbf{z}}=\left(\mathbf{a} \times \mathbf{F}_{\mathrm{R} \|}\right) \hat{\mathbf{z}}>0$ turning the rattleback counterclockwise, as the angle between $\hat{\zeta}$ and $\hat{\mathbf{z}}$ is small during rattleback oscillations. In agreement with Fig. $2 \mathbf{F}_{\mathrm{R} \|} \hat{\xi}$ is positive and it counteracts the top-left inclination of the rattleback when it is touching the supporting surface at point $A$ in Fig. 3a. By symmetry the same situation repeats when the rattleback inclines to the down-right and the resulting torque has again a component contributing to counter clockwise rotation.

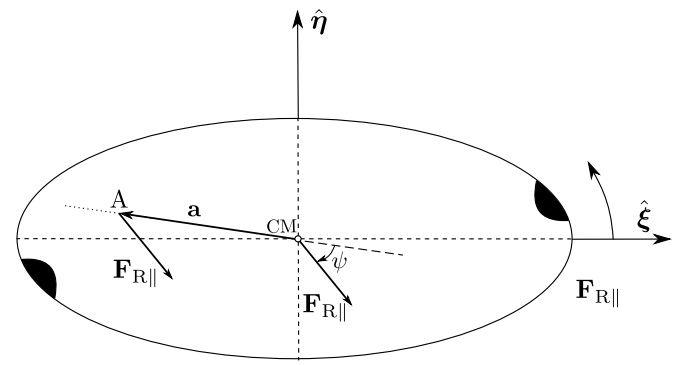

(a)

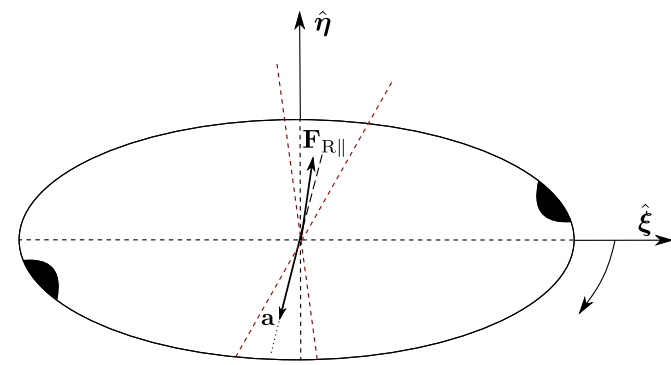

(b)

Figure 3: a) Source of torque in the stable counterclockwise direction (view from top); b) Transversal oscillations generate torque in clockwise direction.

During small energy oscillations of the rattleback the inclination of the plane $(\hat{\boldsymbol{\xi}}, \hat{\boldsymbol{\eta}})$ with respect to plane $(\hat{\mathbf{x}}, \hat{\mathbf{y}})$ is small and picture of relationship between direction of $\mathbf{a}$ and $\mathbf{F}_{\mathrm{R} \|}$ in both planes looks almost the same.

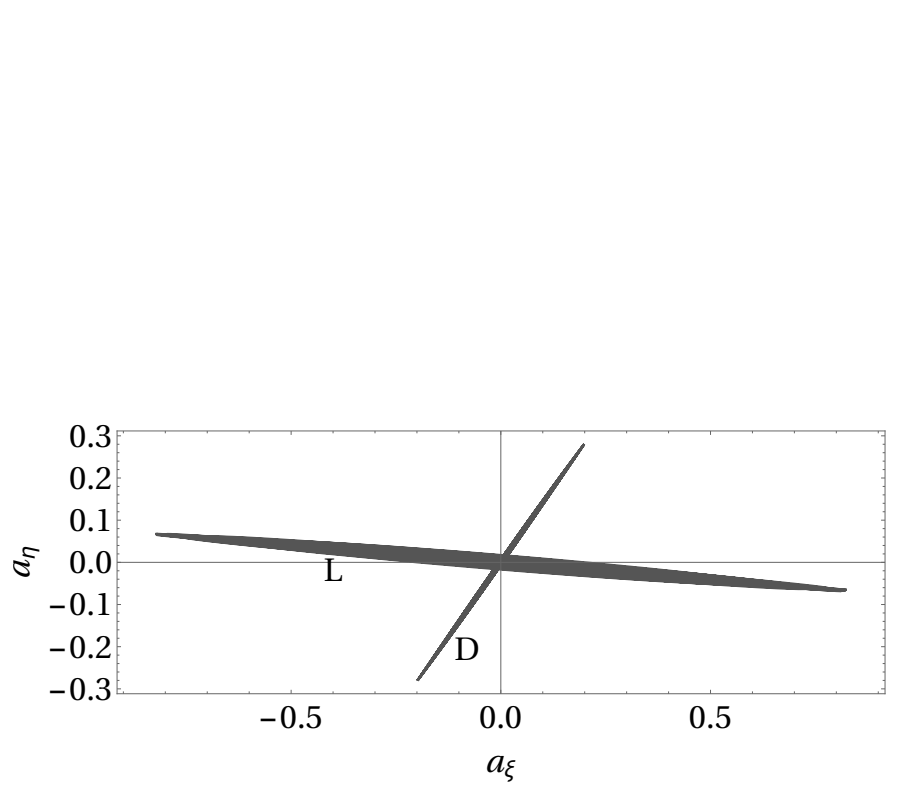

(a) $\mathbf{a} \xi \eta$

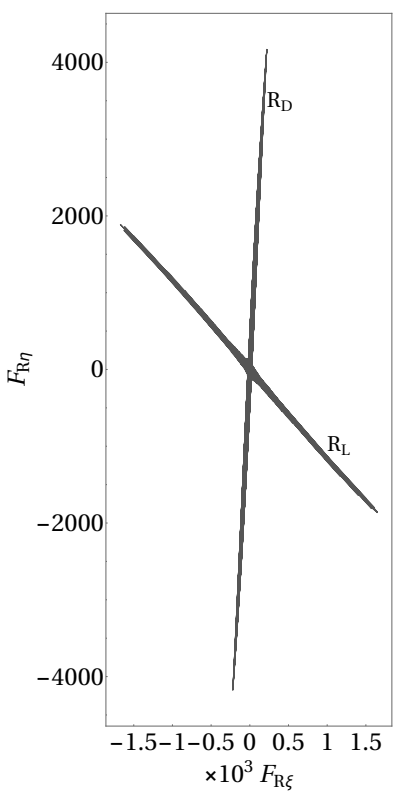

(b) $\mathbf{F}_{\mathrm{R} \|}$

Figure 4: Trajectory of projections of a and $\mathbf{F}_{\mathrm{R}}$ into $(\hat{\boldsymbol{\xi}}, \hat{\boldsymbol{\eta}})$-plane for time $t \in[4.9,46]$. 
Transversal oscillations. When the rattleback is starting with transversal oscillations along short axis of the ellipsoid, then the contact trajectory of the body deflects left in Fig. $3 \mathrm{~b}$ as it is also shown by the shorter downward arm D of the cross-like trajectory $\mathbf{a}_{\xi \eta}(t)$ in Fig. 4a. The corresponding reaction force $\mathbf{F}_{R \|}=\mathbf{F}_{R} \hat{\xi}+\mathbf{F}_{R} \hat{\eta}$ in Fig $3 b$ is directed left of the dashed line extending vector $\mathbf{a}$ and in Fig. $4 \mathrm{~b}$ it is represented by the vertical upward arm $R_{D}$ of the graph $\mathbf{F}_{\mathrm{R} \|}(t)$. This provides a vertical component of the torque $\mathbf{T} \hat{\zeta}=\left(\mathbf{a} \times \mathbf{F}_{\mathrm{R} \|}\right) \hat{\zeta}<0$ pointing towards the plane of support, which is turning the rattleback in the clockwise (!) direction. Indeed the same physical toy rattleback started with transversal oscillations shows a small clockwise rotation effect until it stops to a standstill due to frictional loss of energy not accounted for in this model.

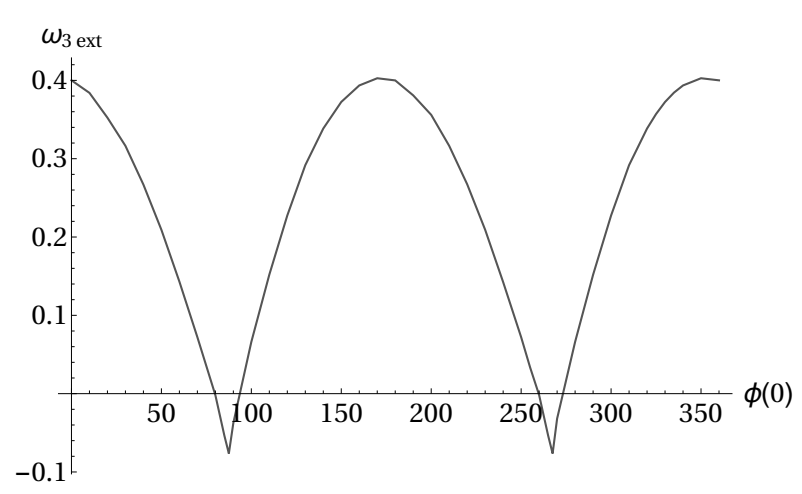

(a) $\omega_{3 \mathrm{ext}}(\phi(0))$

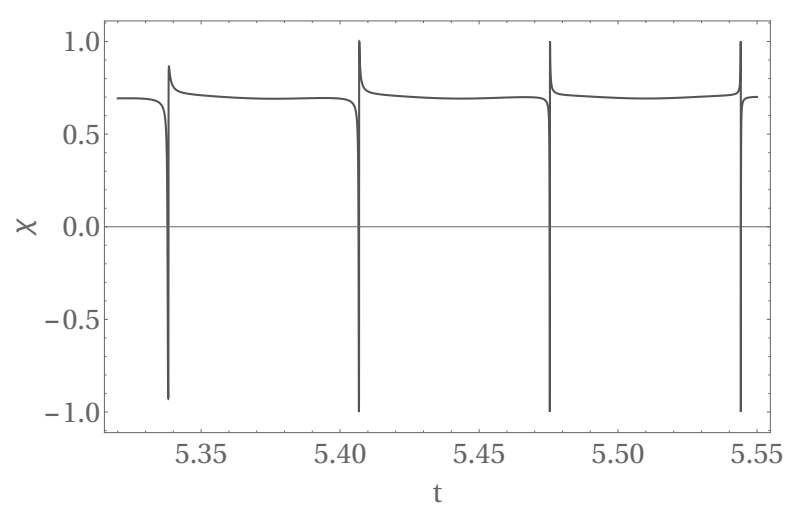

(b) $\chi(t)$

Figure 5: a) Maximal value of $\omega_{3}$ as function of tapping angle $\phi(0) \in\left[0,360^{\circ}\right]$, b) Time evolution of the angle $\chi(t):=\sin \psi(t)$ for $t \in[25.2,25.7]$.

Magnitude of counterclockwise torque. During oscillations the reaction force $\mathbf{F}_{\mathrm{R} \|}(t)$ and the vector $\mathbf{a}(t)$ take simultaneously minimal values when the rattleback is passing close to the static equilibrium. The vertical component of the torque $\mathbf{T} \hat{\zeta}=\left(\mathbf{a} \times \mathbf{F}_{\mathrm{R} \|}\right) \hat{\zeta}>0$ appears to be prevalent positive and oscillates between 0 and maximal value 1400 (see the right $y$ axis in Fig. 6a). What is surprising is that the angle $\psi$ between $\mathbf{a}_{\xi \eta}$ and $\mathbf{F}_{\mathrm{R} \|}$ remains almost constant (see Fig. 5b) with $\chi=\sin \psi=\frac{a_{\xi} F_{R \eta}-a_{\eta} F_{R \xi}}{\sqrt{a_{\xi}^{2}+a_{\eta}^{2}} \sqrt{F_{R \zeta}^{2}+F_{R \eta}^{2}}} \gtrsim 0.7$ for most of the oscillation period except very short time intervals when a is vertical and $\mathrm{CM}$ of the rattleback passes the static equilibrium. Approximate constancy of $\psi$ is visible in Fig. 4 where the arms $\mathrm{L}, \mathrm{D}$ of $\mathbf{a}_{\xi \eta}(t)$ and $\mathrm{R}_{\mathrm{L}}, \mathrm{R}_{\mathrm{D}}$ of $\mathbf{F}_{\mathrm{R} \|}(t)$ are stretched along almost straight lines. Small negative values of the vertical torque $T \hat{\zeta}$ during oscillations in Figs. $6 \mathrm{a}$ and $6 \mathrm{~b}$ may happen only when rattleback passes the static equilibrium. They correspond to sharp negative spikes for $\chi=\sin \psi$ in Fig. $5 b$.

Why is an oscillatory rattleback usually acquiring counter clockwise rotation? As said above a rattleback may acquire rotation in both directions depending on the initial longitudinal or transversal oscillatory initial conditions (IC). So for each such IC there is a neighborhood where $\mathbf{F}_{\mathrm{R} \|}=$ $\mathbf{F}_{R} \hat{\xi}+\mathbf{F}_{R} \hat{\eta}$ is still below (or left) of the dashed line extending vector a in Fig. 3a and Fig. 3b respectively. By continuity there has to be a direction of initial oscillation where $\mathbf{F}_{R \|}=\mathbf{F}_{R} \hat{\xi}+\mathbf{F}_{R} \hat{\eta}$ is pointing along the line of a and the vertical torque is close to zero. This direction separates zones where a rattleback is turning clockwise or counterclockwise.

Dependence of sense of rotation on the angle $\phi(0)$ between $\hat{\xi}$ and the direction of initial tapping oscillation is depicted by graph in Fig 5a showing maximal value of $\omega_{3}$ attained directly after the first reversal. For a rattleback with parameters used in our simulations, discussed in Sec. 4, the region for counterclockwise sense of rotations is very wide as it corresponds to positive parts of curve $\omega_{3 \text { ext }}(\phi(0))$. 


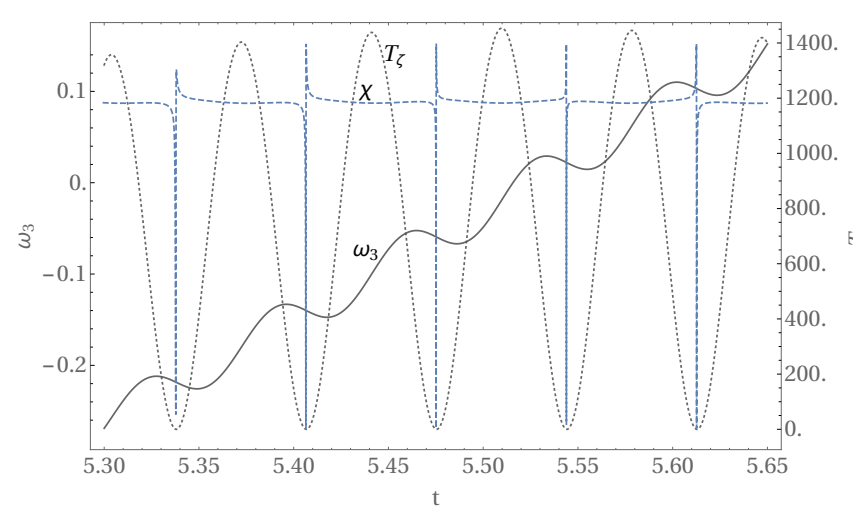

(a) forth reversal

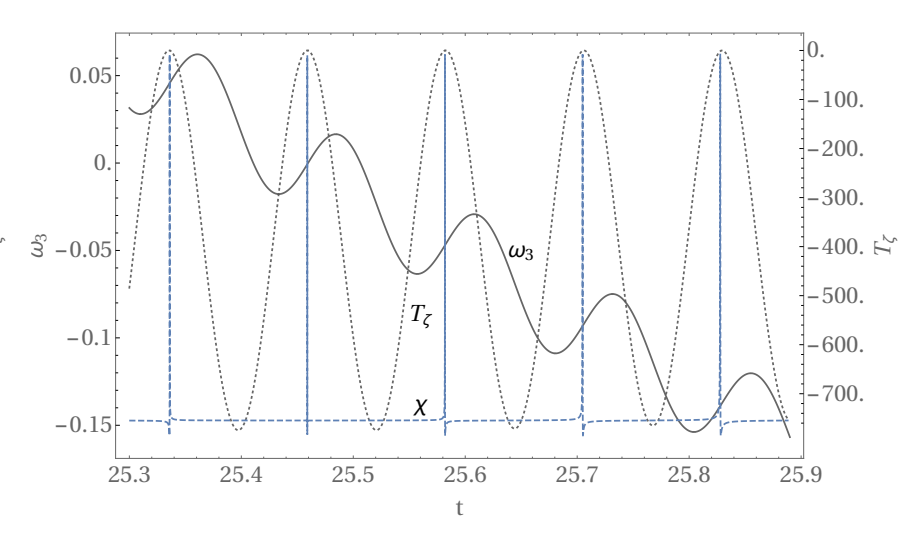

(b) back reversal

Figure 6: Time evolution of $\omega_{3}$ (solid line) and $T_{\zeta}$ (dotted line) over a short time interval close to reversal and back reversal times. Left $y$ axis scales $\omega_{3}$ and right $y$ axis scales $T_{\zeta}$. On both pictures profiles of $\chi(t)$ are marked blue.

Initial angles when the rattleback turns in the clockwise sense of rotation are about $80^{\circ}<\phi(0)<$ $93^{\circ}$ and $260^{\circ}<\phi(0)<273^{\circ}$ when counting from $\hat{\xi}$ axis. These zones are marked by red dotted lines in Fig. 3b. It is not surprising that for typical experimental oscillatory initial conditions, when a toy rattleback is tapped at the long side, it usually starts within the counterclockwise rotational zone (see [15]) and a prefered uni-directional sense of rotations is observed.

Clockwise spinning IC. For understanding reversals of rattleback it is instructive to consider longitudinal oscillatory IC first, but the most surprising counterintuitive effect is the change of direction of rotation when the rattleback is started with the clockwise rotation having here $\omega_{3}(0)=-1$ for IC of type a) in Section 4. With such IC the rattleback first rotates, then starts to wobble and acquires longitudinal oscillations leading to counterclockwise rotation.

So why is a typical rattleback usually reversing from the clockwise rotation although two possible directions of rotation are admissible depending on whether it starts with longitudinal or transversal oscillations? A possible explanation is that a toy rattleback, when it is launched with spin in the clockwise direction is also slightly oscillating. As the angle of counterclockwise oscillatory zone is much wider than for clockwise zone (see Fig. 5a) the rattleback lunched in the clockwise direction is more likely to have sufficient longitudinal oscillations to be a source of the counter clockwise torque transferring energy from rotation to increase longitudinal oscillations and to reverse rotation of the rattleback.

Possibility of multiple reversals. This vectorial discussion explains how a typical toy rattleback may acquire rotation in either direction depending on prevailing type of oscillations. A rattleback with IC of type a) and b) in Section 4, used in our simulations, starts with small energy oscillations due to tipping the axis $\hat{\zeta}$ w.r.t. axis $\hat{\mathbf{z}}$ by $\hat{\mathbf{z}} \hat{\zeta}=\frac{1}{100}$. The first reversal takes place at about $5.5 \mathrm{~s}$ and the back reversal starts at $25.5 \mathrm{~s}$. The long time evolution is quasiperiodic with period of about $50 \mathrm{~s}$ as may be seen in Fig. 10. This picture is consistent with predictions of integrable, truncated rattleback equations introduced by Markeev [16] which seem to describe correctly qualitative global behavior of a rattleback starting with small initial oscillations. A mechanism of change of direction of rotation of a spinning solution, when oscillation are very small, remains unclear. The angle between $\mathbf{a}$ and $\mathbf{F}_{\mathrm{R} \|}$ has to change sign but how it happens is difficult to discern from analysis of simulations.

\section{Rattleback model}

We simulate dynamics of a rattleback toy having a form of homogeneous triaxial half-ellipsoid with semi-axes $b_{1}>b_{2}>b_{3}$ and mass $m_{e}$. To make its mass distribution chiral two material points of 
mass $m_{p}$ are placed at the ellipsoidal edge of the flat top plane of the rattleback at points $r_{0}=\left(x_{0}, y_{0}\right)$, $-r_{0}=\left(-x_{0},-y_{0}\right)$ where $x_{0}$ and $y_{0}$ satisfy the ellipse equation $\frac{x^{2}}{b_{1}^{2}}+\frac{y^{2}}{b_{2}^{2}}=1$.

The homogeneous halv-ellipsoid has center of mass (CM) at distance $\frac{3}{8} b_{3}$ from the top flat surface and principal axes of inertia are directed along the main geometrical axes described by the frame $(\hat{\xi}, \hat{\eta}, \hat{\zeta})$. The main moments of inertia with respect to axes attached to the geometrical center of ellipsoid are $J_{1}, J_{2}, J_{3}$ and the tensor of inertia is diagonal $\mathbb{I}_{e}=\operatorname{diag}\left(J_{1}, J_{2}, J_{3}\right)$, where $J_{1}=\frac{m_{e}}{5}\left(b_{2}^{2}+b_{3}^{2}\right), J_{2}=$ $\frac{m_{e}}{5}\left(b_{3}^{2}+b_{1}^{2}\right), J_{3}=\frac{m_{e}}{5}\left(b_{1}^{2}+b_{2}^{2}\right)$. Each of material points of mass $m_{p}$ placed at point $r_{0}$ or $-r_{0}$ has with respect to the same axes non-diagonal tensor of inertia

$$
\mathbb{I}_{m}=m_{p}\left(\begin{array}{ccc}
y_{0}^{2} & -x_{0} y_{0} & 0 \\
-x_{0} y_{0} & x_{0}^{2} & 0 \\
0 & 0 & x_{0}^{2}+y_{0}^{2}
\end{array}\right) .
$$

Adding 2 extra masses $m_{p}$ makes the center of mass to be at distance $d=\frac{3}{8} \frac{m_{e} b_{3}}{m_{e}+2 m_{p}}$ from the top flat surface and the matrix of inertia with respect to the main geometrical axes attached to $\mathrm{CM}$ is

$$
\begin{aligned}
& \mathbb{I}=\left(\begin{array}{ccc}
I_{11} & -I_{12} & 0 \\
-I_{12} & I_{22} & 0 \\
0 & 0 & I_{33}
\end{array}\right) \\
& I_{11}=\frac{m_{e}}{5}\left(b_{2}^{2}+b_{3}^{2}\right)+2 m_{p} y_{0}^{2}-\frac{9}{64} \frac{m_{e}^{2} b_{3}^{2}}{m_{e}+2 m_{p}}, \quad I_{12}=2 m_{p} x_{0} y_{0} \\
& I_{22}=\frac{m_{e}}{5}\left(b_{3}^{2}+b_{1}^{2}\right)+2 m_{p} x_{0}^{2}-\frac{9}{64} \frac{m_{e}^{2} b_{3}^{2}}{m_{e}+2 m_{p}}, \quad I_{33}=\frac{m_{e}}{5}\left(b_{1}^{2}+b_{2}^{2}\right)+2 m_{p}\left(x_{0}^{2}+y_{0}^{2}\right) .
\end{aligned}
$$

Presence of masses $m_{p}$ rotates principal axes of inertia left $\hat{\mathbf{1}}=\hat{\boldsymbol{\xi}} \cos \delta+\hat{\boldsymbol{\eta}} \sin \delta, \hat{\mathbf{2}}=-\hat{\boldsymbol{\xi}} \sin \delta+\hat{\boldsymbol{\eta}} \cos \delta$, $\hat{\mathbf{3}}=\hat{\zeta}$ by angle

$\delta=\frac{1}{2} \arctan \left(\frac{2 I_{12}}{I_{22}-I_{11}}\right)=\frac{1}{2} \arctan \left(\frac{20 m_{p} x_{0} y_{0}}{m_{e}\left(b_{1}^{2}-b_{2}^{2}\right)+10 m_{p}\left(x_{0}^{2}-y_{0}^{2}\right)}\right)$ as in Fig. 1 and matrix of inertia takes the form [9]

$$
\mathbb{I}=\left(\begin{array}{ccc}
I_{1} \cos ^{2} \delta+I_{2} \sin ^{2} \delta & \left(I_{1}-I_{2}\right) \sin \delta \cos \delta & 0 \\
\left(I_{1}-I_{2}\right) \sin \delta \cos \delta & I_{2} \cos ^{2} \delta+I_{1} \sin ^{2} \delta & 0 \\
0 & 0 & I_{3}
\end{array}\right),
$$

where $I_{1}, I_{2}, I_{3}$ are eigenvalues of $\mathbb{I}$ given in (3.1).

In our simulations we consider a halv-ellipsoid with semi-axes $b_{1}=7.5 \mathrm{~cm}, b_{2}=2 \mathrm{~cm}, b_{3}=1.5$ $\mathrm{cm}$ having the mass $m_{e}=18 \mathrm{~g}$ and two mass points $m_{p}=3 \mathrm{~g}$ localized at $r_{0}=(6,6 / 5)$ and $-\boldsymbol{r}_{0}$, gravitational constant equals to $g=981 \mathrm{~cm} / \mathrm{s}^{2}$. The tensor of inertia with respect to geometric axes $(\hat{\xi}, \hat{\eta}, \hat{\zeta})$ is then

$$
\mathbb{I}=\left(\begin{array}{ccc}
\frac{343917}{12800} & -\frac{216}{5} & 0 \\
-\frac{216}{5} & \frac{1081161}{2560} & 0 \\
0 & 0 & \frac{22077}{50}
\end{array}\right) \text {. }
$$

It has eigenvalues $I_{1} \approx 22.20 \mathrm{~g} \mathrm{~cm}^{2}, I_{2} \approx 426.99 \mathrm{~g} \mathrm{~cm}^{2}, I_{3} \approx 441.54 \mathrm{~g} \mathrm{~cm}^{2}$ and $\delta \approx 6.16^{\circ}$.

It is convenient to use equations of motion represented in the body fixed frame $(\hat{\xi}, \hat{\eta}, \hat{\zeta})$. Then equations (2.2) take the form [5, 9, 7]

$$
\begin{aligned}
& \mathbb{I} \dot{\boldsymbol{\omega}}+m \mathbf{a} \times(\dot{\boldsymbol{\omega}} \times \mathbf{a})+m \mathbf{a} \times(\boldsymbol{\omega} \times \dot{\mathbf{a}})=\mathbb{I} \boldsymbol{\omega} \times \boldsymbol{\omega}+m(\boldsymbol{\omega}, \mathbf{a}) \boldsymbol{\omega} \times \mathbf{a}+m g \mathbf{a} \times \gamma, \\
& \dot{\gamma}=\boldsymbol{\omega} \times \gamma
\end{aligned}
$$

where $\gamma$ is a unit vector pointing vertically upwards and $\mathbb{I}$ is given by Eq. (3.2). If the angular momentum of the body with respect to the contact point is $\mathbf{M}=\mathbb{I} \boldsymbol{\omega}+m \mathbf{a} \times(\boldsymbol{\omega} \times \mathbf{a})$, then these equations can be 
written in a more compact form $\dot{\mathbf{M}}=\mathbf{M} \times \boldsymbol{\omega}+m \dot{\mathbf{a}} \times(\boldsymbol{\omega} \times \mathbf{a})+m g \mathbf{a} \times \gamma, \dot{\gamma}=\boldsymbol{\omega} \times \gamma$ (see [9, 7]). This system has two first integrals $F_{1}=\langle\gamma, \gamma\rangle, F_{2}=\frac{1}{2}\langle\mathbf{M}, \boldsymbol{\omega}\rangle-m g\langle\mathbf{a}, \gamma\rangle$, which are used for checking accuracy of calculations. Since the direction of the unit vector $\gamma$ at the contact point $A$ is connected to the vector a pointing from the geometrical center of a whole tri-axial ellipsoid $F(\mathbf{a})=\frac{r_{1}^{2}}{b_{1}^{2}}+\frac{r_{2}^{2}}{b_{2}^{2}}+\frac{r_{3}^{2}}{b_{3}^{2}}-1=0$ the connecting formula $\gamma=-\frac{\nabla F(\mathbf{a})}{|\nabla F(\mathbf{a})|}$, allows for expressing a in terms of $\gamma[9,7]$. Our vector $\mathbf{a}=\left(a_{1}, a_{2}, a_{3}\right)$ pointing from $\mathrm{CM}$ toward the contact point $\mathrm{A}$, after taking into account displacement of the mass center for the halv-ellipsoid due to adding two mass points $m_{p}$, has coordinates

$a_{1}=-\frac{b_{1}^{2} \gamma_{1}}{\sqrt{b_{1}^{2} \gamma_{1}^{2}+b_{2}^{2} \gamma_{2}^{2}+b_{3}^{2} \gamma_{3}^{2}}}, a_{2}=-\frac{b_{2}^{2} \gamma_{2}}{\sqrt{b_{1}^{2} \gamma_{1}^{2}+b_{2}^{2} \gamma_{2}^{2}+b_{3}^{2} \gamma_{3}^{2}}}, a_{3}=-\frac{b_{3}^{2} \gamma_{3}}{\sqrt{b_{1}^{2} \gamma_{1}^{2}+b_{2}^{2} \gamma_{2}^{2}+b_{3}^{2} \gamma_{3}^{2}}}+\frac{3}{8} \frac{m_{e} b_{3}}{m_{e}+2 m_{p}}$.

Equations of motion (3.3) have two stationary solutions:

- 1) $\boldsymbol{\omega}=(0,0, \Omega)$ and $\gamma=(0,0,1)$ and

- 2) $\boldsymbol{\omega}=(0,0, \Omega)$ and $\gamma=(0,0,-1)$.

One can check their linear stability by applying the Routh-Hurwitz criterion to the Jacobi matrices of linearisations around these solutions. This criterion gives necessary and sufficient conditions for all characteristic eigenvalues of the analyzed matrix to have negative real parts. For values of principal moments of inertia and masses considered in our rattleback model the solution 1) is always linearly unstable. Necessary stability conditions obtained from the Routh-Hurwitz criterion for solution 2) are the following:

$$
\begin{aligned}
& \Omega \sin (2 \delta)>0, \quad-1402830+2369 \Omega^{2}>0, \\
& 158745465780+252407893 \Omega^{2}-2090 \sqrt{5057209}\left(20928+41 \Omega^{2}\right) \cos (2 \delta)>0, \\
& 8704314654750+144046235355 \Omega^{2}+252407893 \Omega^{4}-2090 \sqrt{5057209} \Omega^{2}\left(20928+41 \Omega^{2}\right) \cos (2 \delta)>0 .
\end{aligned}
$$

For all $-\pi \leq \delta \leq \pi$ and $\Omega^{2} \geq 0$ the third and the fourth inequalities are satisfied and the first two give $\delta \in\left[-\pi,-\frac{\pi}{2}\right] \cup\left[0, \frac{\pi}{2}\right]$ and $\Omega>3 \sqrt{\frac{155870}{2369}} \approx 24.3344$ or $\delta \in\left[-\frac{\pi}{2}, 0\right] \cup\left[\frac{\pi}{2}, \pi\right]$ and $\Omega<-3 \sqrt{\frac{155870}{2369}} \approx$ -24.3344. The solution 2) describes an ellipsoid turned upside down with CM above geometrical center, which is irrelevant for this study of low energy spinning and oscillating solutions with $|\omega| \leq 1$. Thus both spinning solutions $\omega=(0,0, \Omega)$ with $\Omega= \pm 1$ are linearly unstable in simulations of Section 4 .

\section{Numerical simulations}

The purpose of numerical simulations is studying reversals of a toy rattleback discussed in Sec. 2 and Sec. 3 by starting it with initial conditions corresponding to gentle rotating in the clockwise direction or to tapping the rattleback on the side as for typical demonstrations. All simulations were made using software Mathematica with working precision at least 12 so that precision of 12 digits has been maintained during internal computations. We have chosen 2 types of initial conditions (IC) corresponding to:

a) rotating the rattleback clockwise with initial angular velocity $\omega=\left(0,0, \omega_{3}=-1\right)$ at small inclination angle $\gamma=\left(0, \frac{1}{1000}, \frac{3 \sqrt{111111}}{1000}\right)$ sufficient to start small initial longitudinal oscillations that grow in time. The rotational velocity $\omega_{3}=-1$ corresponds to small energy $E_{\text {rot }}=25604$. It has the same order of magnitude as energy of tapping IC in b) with $24426 \leq E_{\text {tapping }} \leq 25385$.

b) tapping the rattleback at elliptic edge points at different angles $\phi(0)$ measured from $\hat{\xi}$-axis, with initial inclination satisfying $\hat{\mathbf{z}} \hat{\zeta}=\sqrt{\gamma_{1}^{2}+\gamma_{2}^{2}}=1 / 100$ and $\boldsymbol{\omega}=\mathbf{0}$.

The values of $\omega_{3}$ are small thus both stationary solutions 1) and 2) of Section 3 are linearly unstable. All tested solutions exhibit quasiperiodic type of behavior with multiple reversals and lengths of period 
inversely related to initial energy. This is in agreement with different types of dynamic regimes discussed in [8]. In Fig. 7 we show time evolution of $\omega_{3}$ for initial condition a) with various initial values of $\omega_{3}$ and of energy $E$. One can also note various regimes of dynamics that are a little different from those presented in Fig. 4 in [17]. With increasing energy the rattleback's motion is losing its regular quasi-periodic character.

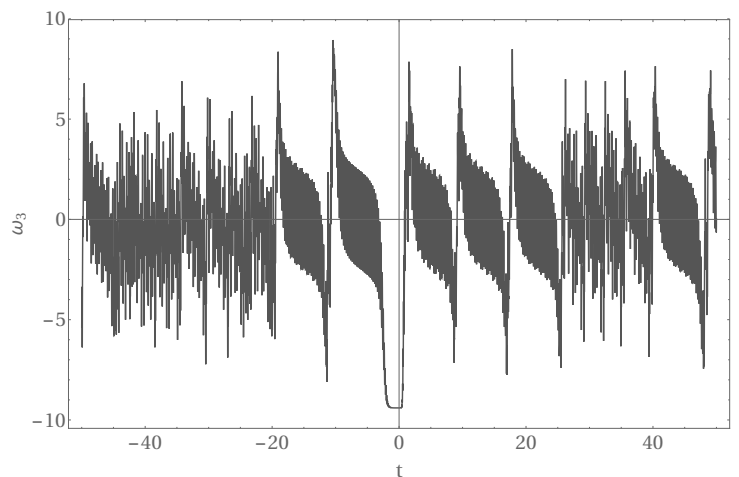

(a) $\omega_{3}(0)=-9.4, E=44890.6$

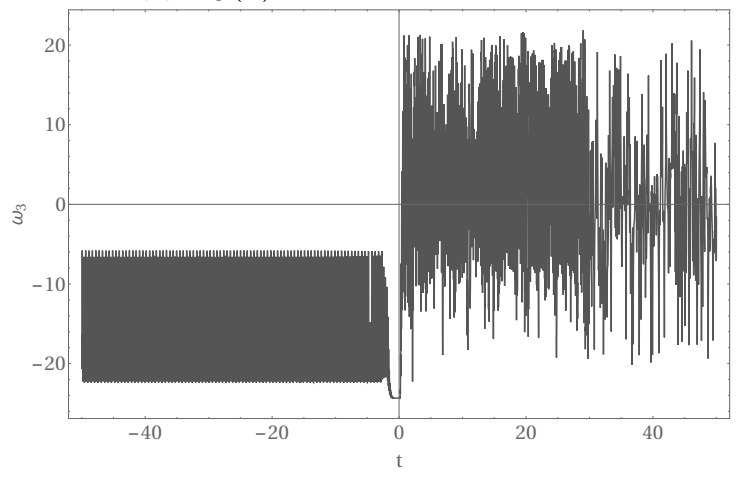

(c) $\omega_{3}(0)=-3 \sqrt{\frac{155870}{2369}}, E=156115$

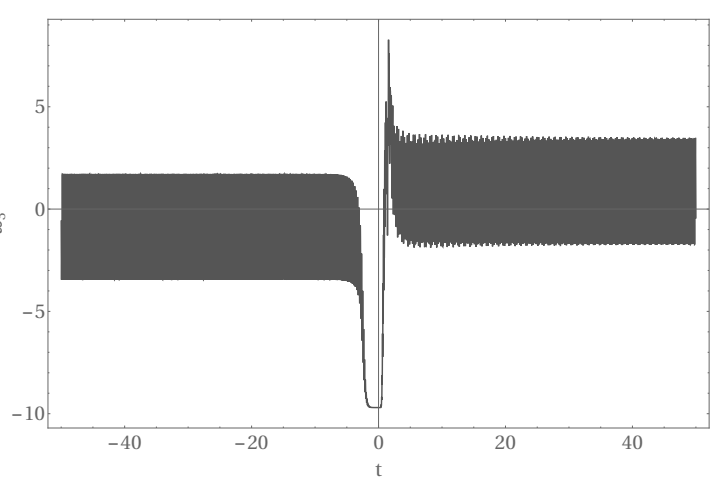

(b) $\omega_{3}(0)=-9.7, E=46155.7$

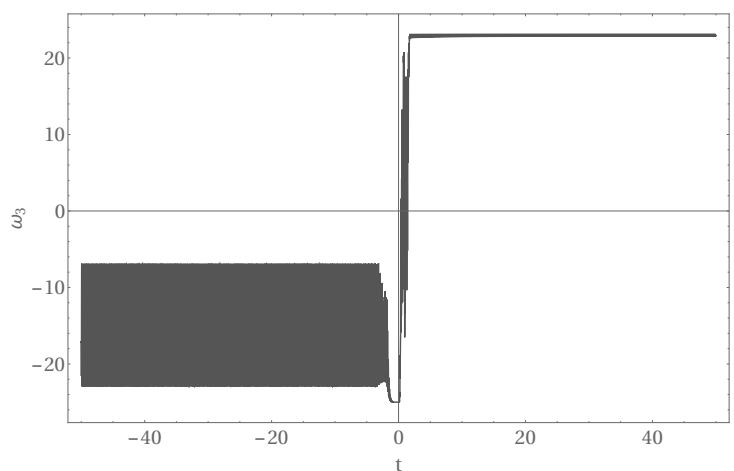

(d) $\omega_{3}(0)=-25, E=163365$

Figure 7: Dependence of $\omega_{3}$ on time for various values of energy.

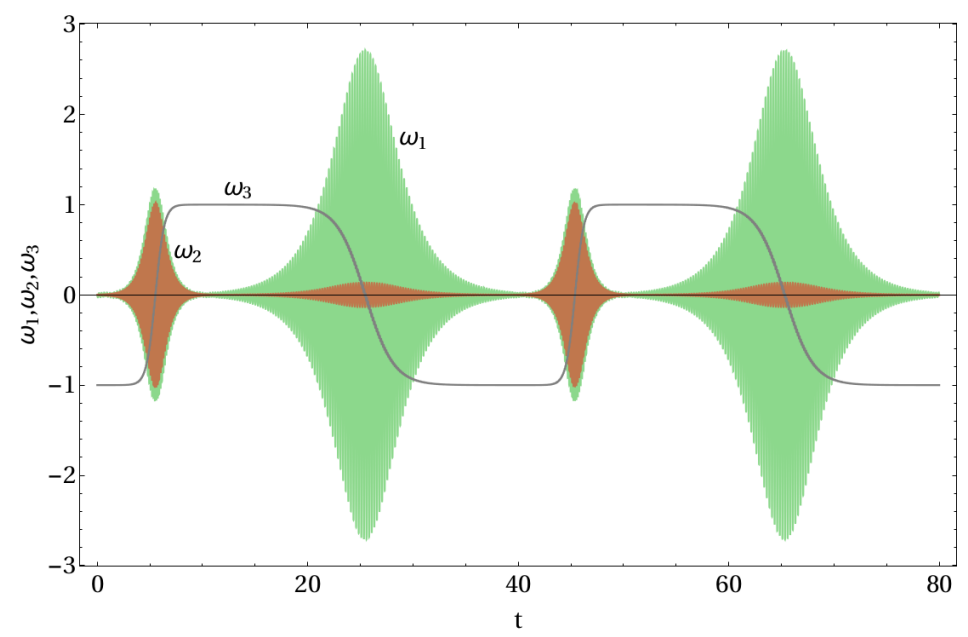

Figure 8: Time evolution of components $\omega_{i}$ over a time interval of 4 reversals. 
Rotating initial conditions $\omega=\left(0,0, \omega_{3}=-1\right), \gamma=(0,0.001,0.003 \cdot \sqrt{111111})$. Fig. 8 shows $\hat{\xi}, \hat{\eta}, \hat{\zeta}$ components of angular velocity $\omega$ over a time interval of 4 reversals of the rattleback when $\omega_{3}$ changes from -1 to 1 and back. A single reversal cycle takes about $40 \mathrm{~s}$. During the counterclockwise reversal at about $5.5 \mathrm{~s}$ angular velocities $\omega_{2}$ (brown) and $\omega_{1}$ (green) oscillate rapidly within the shaded envelope as marked in Fig. 8. During the first back reversal at about $25.5 \mathrm{~s}$ transversal oscillations are essential in creating the relevant clockwise acting torque as discussed in Sec. 2.

Enlarged views of $\omega_{1}, \omega_{2}$ during both reversal time moments, when $\omega_{3} \approx 0$ are shown in Figs. 9a and $9 \mathrm{~b}$. When longitudinal oscillations are turning rattleback counterclockwise frequencies of $\omega_{1}, \omega_{2}$ are synchronized and have the same size (Fig. 9a). During transversal oscillations $\omega_{1}$ is an order of magnitude larger than $\omega_{2}$ and has an opposite sign (Fig. 9b).

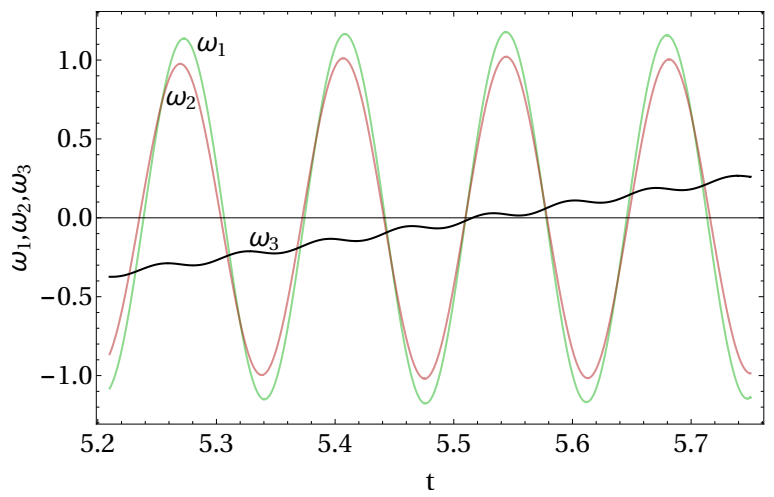

(a) forth reversal

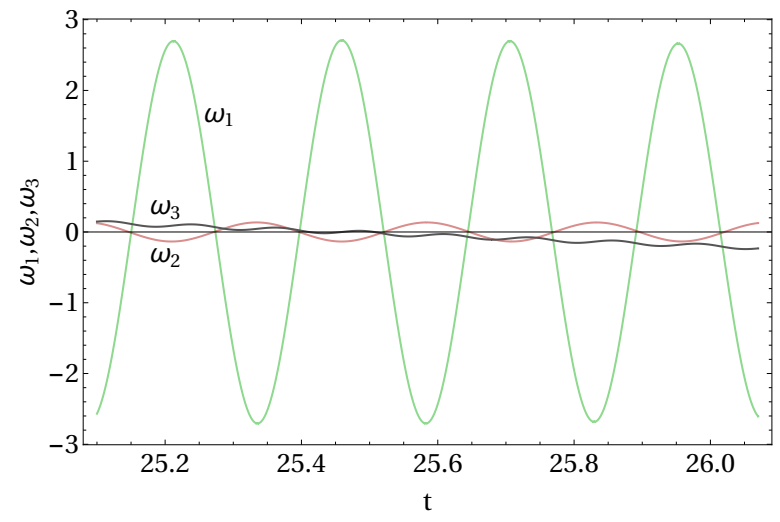

(b) back reversal

Figure 9: Components $\omega_{i}$ during rattleback reversals.

Graph in Fig 10 shows that the vertical component of torque $T_{\hat{\mathbf{z}}}$ becomes large during the reversal phase close to $t=5.5 \mathrm{~s}$ when the rattleback is performing longitudinal oscillations with large $\omega_{2}$. Values of $T_{\hat{\mathbf{z}}}$ are positive during the first counterclockwise reversal and are negative for the back reversal at $t=25.5 \mathrm{~s}$ when the rattleback performs mainly transversal oscillations as can be seen from relative magnitude of $\omega_{1}$ and $\omega_{2}$ in Fig 8.

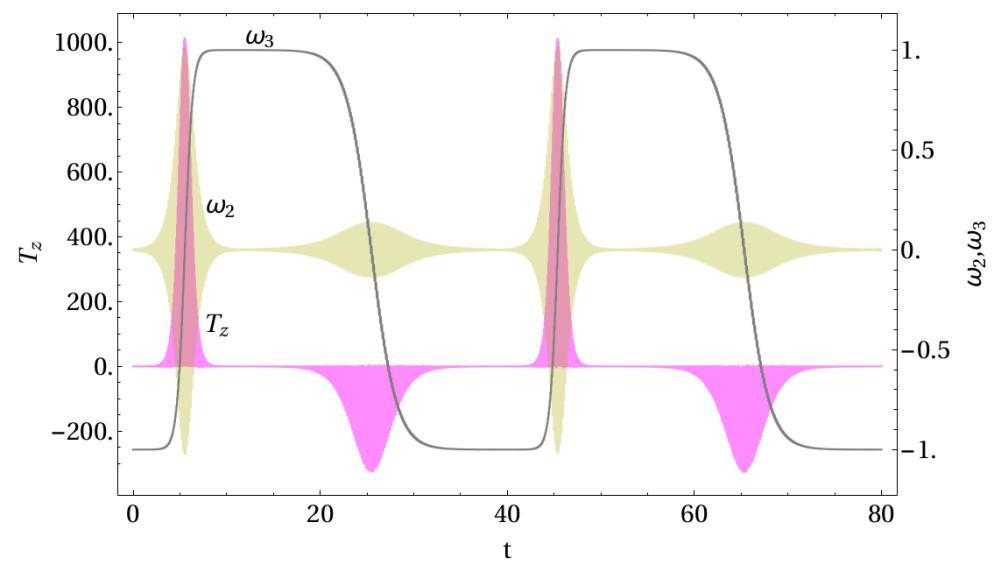

Figure 10: Time evolution of vertical component of torque $T_{z}=\mathbf{T} \hat{\mathbf{z}}$. Left $y$ axis scales $T_{z}$ and right $y$ axis scales $\omega_{2}$ and $\omega_{3}$.

Fig. 11a shows enlarged view of the torque $T_{\hat{\mathbf{z}}}$ over short period of time $t \in[4.7,5.85]$, when $\omega_{3} \approx 0$ and shows individual oscillations of $T_{\hat{\mathbf{z}}}$ in vicinity of maximum of the envelope curve of $T_{\hat{\mathbf{z}}}$ in Fig. 10. Minimal values of torque $T_{\hat{\mathbf{z}}}$ coincide with maximal positive and maximal negative values of $\omega_{2}$ when $\mathrm{CM}$ of the oscillating rattleback is passing close to the static equilibrium position. The maximal values 


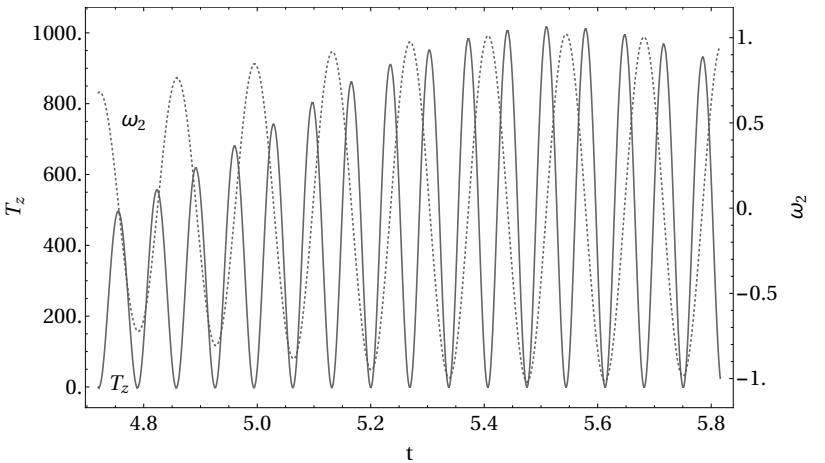

(a) component $T_{z}$

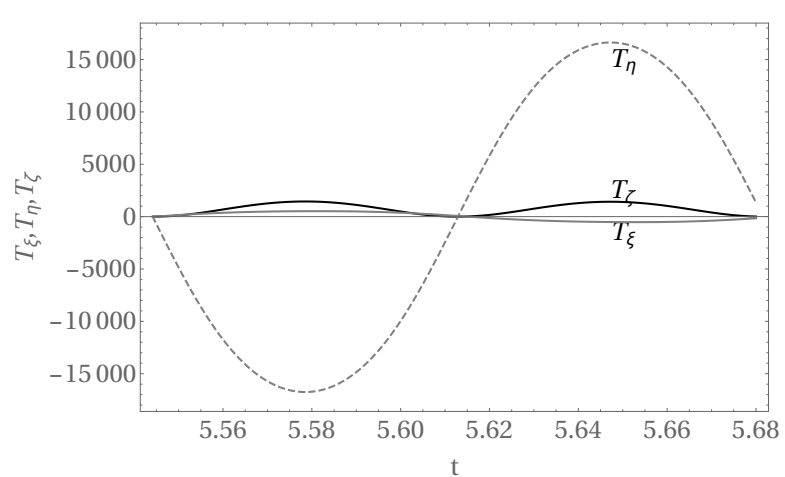

(b) components $T_{\xi}, T_{\eta}$ and $T_{\zeta}$

Figure 11: Time evolution of components of $\mathbf{T}=\mathbf{a} \times \mathbf{F}_{\mathrm{R}}$ for a short time interval close to reversal time $t \approx 5.5 \mathrm{~s}$. In Fig. 11a left $y$ axis scales $T_{z}$ and right $y$ axis scales $\omega_{2}$.

of $T_{\hat{\mathbf{z}}}$ coincide with oscillation reversal points when $\omega_{2}$ is changing its sign. In Fig. 11a the torque $T_{\hat{\mathbf{z}}}$ varies between positive values of about 1000 and values close to 0 when the rattleback passes its equilibrium position. There is no reason to assume that $T_{\hat{\mathbf{z}}}$ is always positive during the reversal. Only at certain few minimum points $T_{\hat{\mathbf{z}}}$ acquires small negative values that can be detected in Fig. $5 \mathrm{~b}$ for

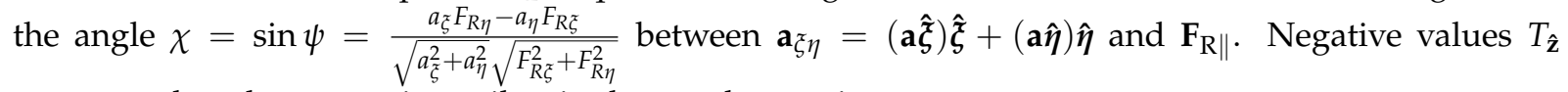
correspond to short negative spikes in the graph $\chi=\sin \psi$.

Relative magnitude of all components of torque over single period of oscillations for $t \in[5.55,5.68]$ is plotted in Fig. 11b. The transversal torque $T_{\eta}$ rocking the rattleback in the longitudinal direction is an order of magnitude larger than the remaining ones.

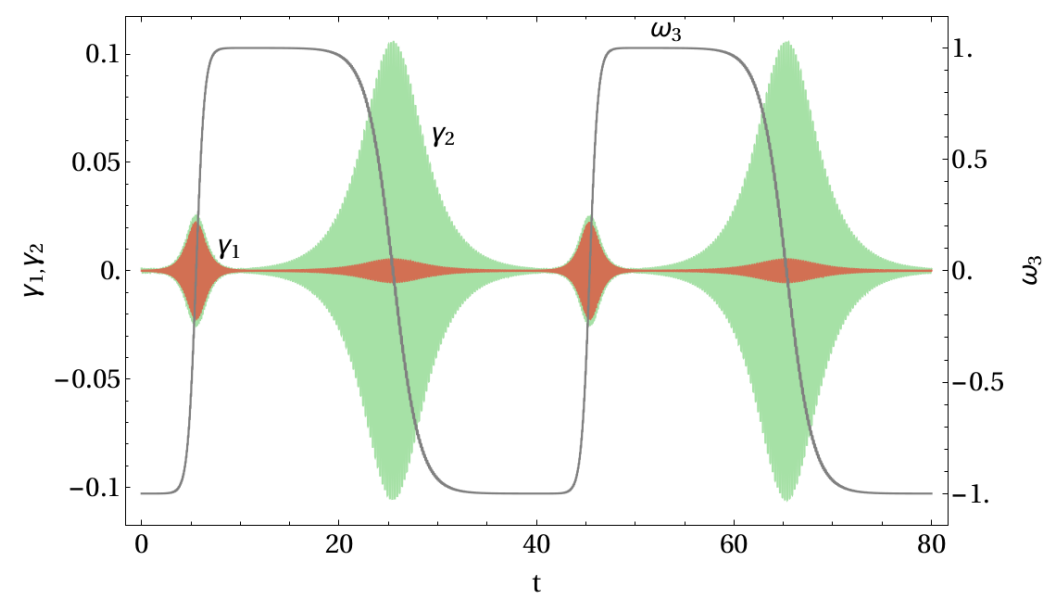

Figure 12: Time evolution of components $\gamma_{1}, \gamma_{2}$ and $\omega_{3}$. Left $y$ axis scales $\gamma_{1}, \gamma_{2}$ and the right $y$ axis scales $\omega_{3}$

The magnitude of cosines describing inclination of vector $\gamma=\left(\gamma_{1}, \gamma_{2}, \gamma_{3}\right)$ in Fig. 12 are also correlated with the reversal and back reversal phases of rattleback motion. The maximum of $\gamma_{2}$ for transversal inclination of rattleback is much larger than $\gamma_{1}$ as the mid-axis $b_{2}=2 \mathrm{~cm}$ is shorter than $b_{1}=7.5 \mathrm{~cm}$ and inclination of rattleback has to be much deeper to give similar potential energy as during the longitudinal oscillations. 


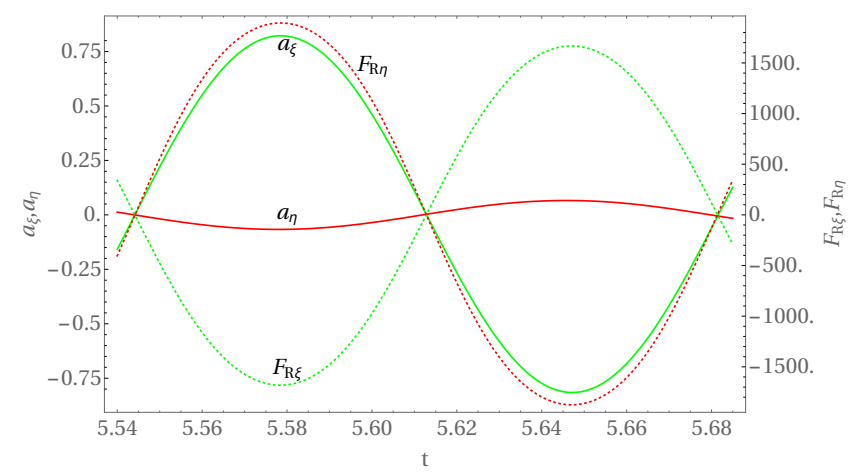

Figure 13: Components of $\mathbf{a}$ and $\mathbf{F}_{\mathrm{R}}$ during reversal. Left $y$ axis scales $a_{\xi}, a_{\eta}$ and right scales $F_{R \xi}, F_{R \eta}$.

Dynamical behaviour of $a_{\xi}(t), a_{\eta}(t), F_{R \xi}(t), F_{R \eta}(t)$ is given in Fig. 13 where we see what happens over a single period of oscillation close to $t=5.5 \mathrm{~s}$. Trajectories of $\mathbf{a}_{\xi \eta}(t), \mathbf{F}_{\mathrm{R} \|}(t)$ are plotted in Fig. 4a and Fig. $4 \mathrm{~b}$. They show that $\mathbf{a}_{\xi \eta}(t)$ oscillate along an almost straight interval during the forth reversal and along a shorter inclined interval during back reversal. Altogether $\mathbf{a}_{\xi \eta}(t)$ fills cross-shaped contour in Fig. 4a. The cross-shaped contour filled up by $\mathbf{F}_{\mathrm{R} \|}(t)$ in Fig. $4 \mathrm{~b}$ shows that the angle $\psi$ between $\mathbf{a}_{\xi \eta}$ and $\mathbf{F}_{\mathrm{R} \|}$ is almost constant during the forth reversal and back reversal respectively.

The energy of rattleback has most transparent form in the $(\hat{\mathbf{1}}, \hat{\mathbf{2}}, \hat{\mathbf{3}})$ frame as the tensor of of inertia is diagonal and the rotational kinetic energy is $E_{\mathrm{rot}}=E_{\mathrm{r} 1}+E_{\mathrm{r} 2}+E_{\mathrm{r} 3}=\left(\frac{1}{2} I_{1} \omega_{1}^{\prime 2}\right)+\left(\frac{1}{2} I_{2} \omega_{2}^{\prime 2}\right)+\left(\frac{1}{2} I_{3} \omega_{3}^{2}\right)$, where $\omega_{1}^{\prime}=\cos \delta \omega_{1}+\sin \delta \omega_{2}, \omega_{2}^{\prime}=-\sin \delta \omega_{1}+\cos \delta \omega_{2}$. The remaining contributions are from the adjusted potential energy $E_{\mathrm{p}}=-m g\langle\mathbf{a}, \gamma\rangle$, and the translational energy of $\mathrm{CM} E_{\mathrm{CM}}=\frac{m}{2} \dot{\mathbf{s}}^{2}=\frac{m}{2}(\mathbf{a} \times \boldsymbol{\omega})^{2}$.

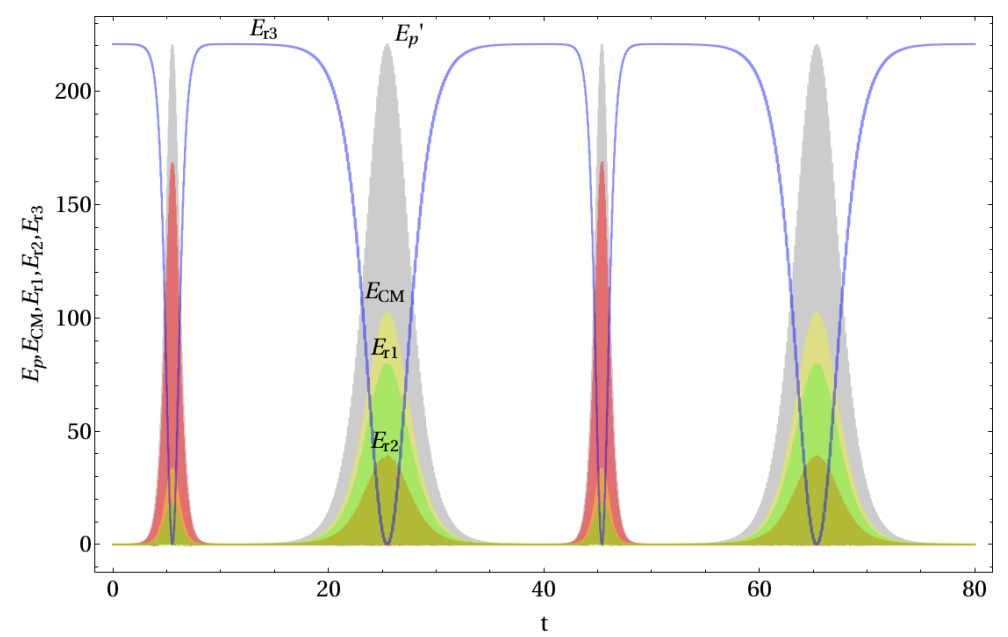

Figure 14: Time evolution of energy components: $E_{\mathrm{p}}^{\prime}(t)=E_{\mathrm{p}}(t)-25383.4, E_{\mathrm{CM}}(t), E_{\mathrm{r} 1}(t), E_{\mathrm{r} 2}(t)$ and $E_{\mathrm{r} 3}(t)$ for $t \in[0,80]$.

Figures 14 and 15 illustrate the energy exchange between the oscillational, rotational and potential modes on long reversal time scale and short oscillation time scale, respectively. Values of energy components visually add up to the constant value equal to $E_{\mathrm{p}}^{\prime}$ in Fig. 15. 


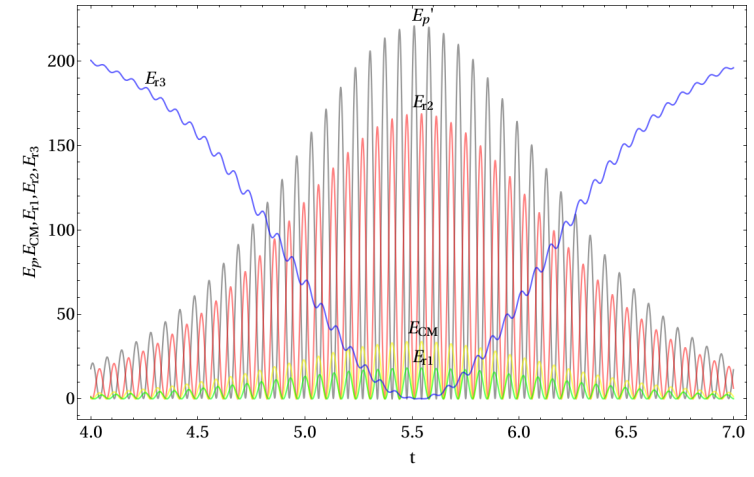

(a) forth reversal

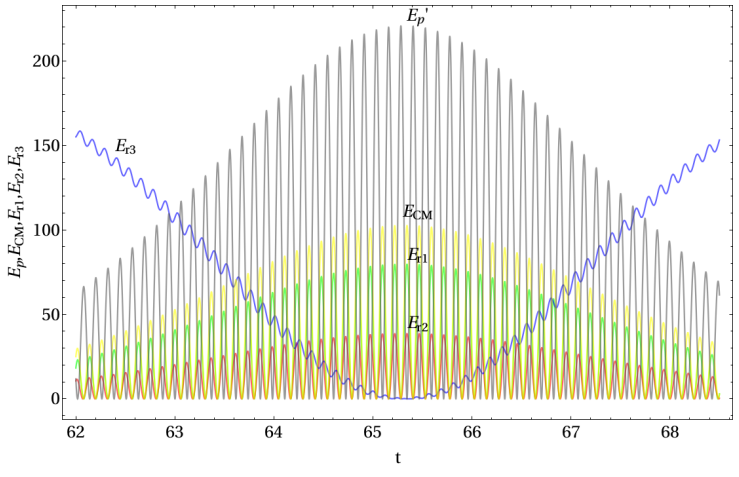

(b) back reversal

Figure 15: Time evolution of energy components: $E_{\mathrm{p}}^{\prime}(t)=E_{\mathrm{p}}(t)-25383.4, E_{\mathrm{CM}}(t), E_{\mathrm{r} 1}(t), E_{\mathrm{r} 2}(t)$ and $E_{\mathrm{r} 3}(t)$ during reversals.

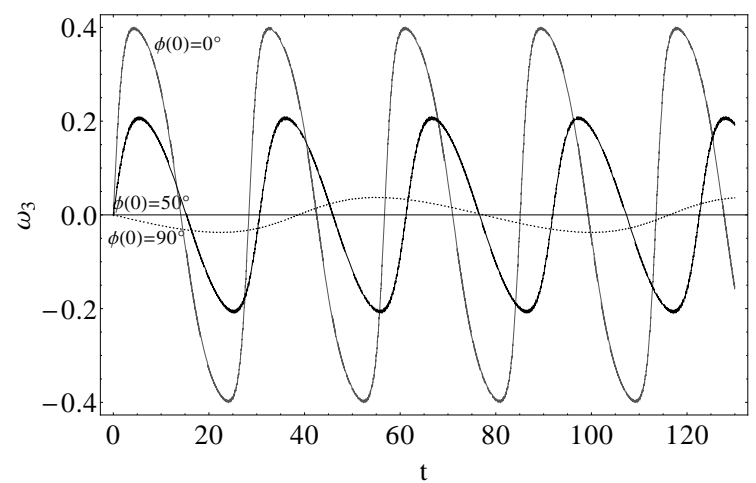

(a) $\phi(0)=0^{\circ}, 50^{\circ}, 90^{\circ}$

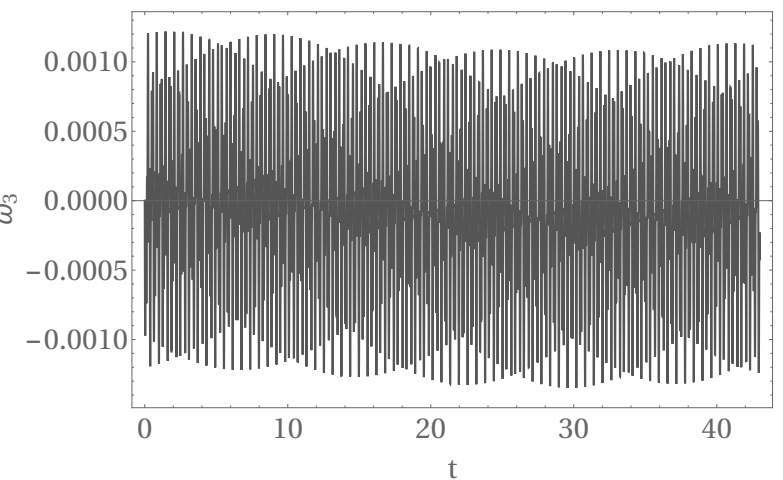

(b) $\phi(0)=79.14^{\circ}$

Figure 16: Reversals of $\omega_{3}(t)$ for several tapping angles $\phi(0)$.

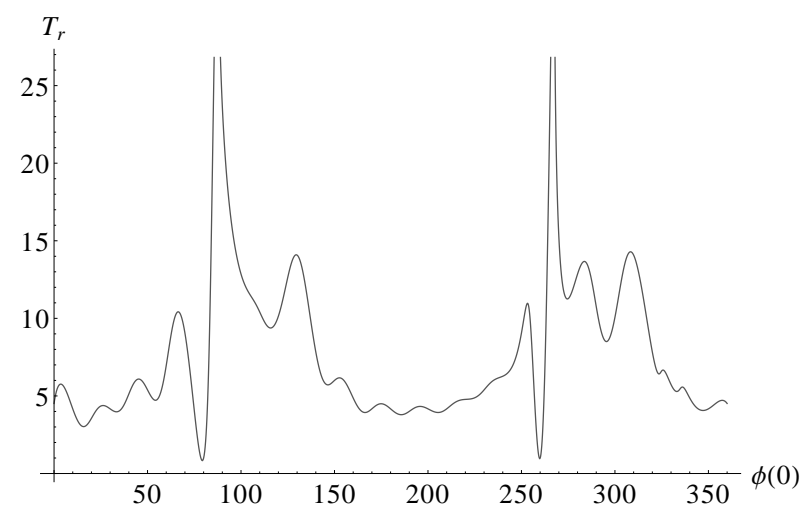

Figure 17: Dependence of the first reversal time $T_{r}$ on tapping angle $\phi(0) \in[0,2 \pi]$.

The graph in Fig. 5a illustrates how the maximal rotational velocity $\omega_{3}$ depends on initial tapping angle $\phi(0)$ when a rattleback is inclined having $\omega(0)=\mathbf{0}$ and $\sqrt{\gamma_{1}^{2}+\gamma_{2}^{2}}=1 / 100$, where $\gamma_{1}=\frac{1}{100} \cos \phi(0)$, $\gamma_{2}=\frac{1}{100} \sin \phi(0), \phi(0) \in[0,2 \pi]$. For $79.14^{\circ}<\phi(0)<93.29^{\circ}$ and $259.14^{\circ}<\phi(0)<273.29^{\circ}$ a tapped rattleback acquires clockwise rotation while for the remaining, and much wider range of angles between 
$0^{\circ}$ and $360^{\circ}$ it starts to turn counterclockwise from the initial oscillations. A rattleback starting at angles close to the border values $79.14^{\circ}, 93.29^{\circ}$ acquires very small velocity $\omega_{3}(t)$, which is oscillating wildly between positive and negative values so that there is no preferred sense of rotation of the rattleback in Fig. 16b. Fig. 16a shows how the first reversal time and maximal value of $\omega_{3}$ depends on tapping angle $\phi(0)$ of oscillatory IC. The maximal value of $\omega_{3}$ during first reversal decreases for increasing values of $\phi(0)$ but there is no such regularity for the time $T_{r}$ of first reversal when $\phi(0)$ increases (Fig. 17).

\section{Conclusions}

We have discussed an intuitive way of understanding unidirectional motion of an ellipsoidal shaped toy rattleback by using frictionless Newton equations for a rigid body rolling without sliding on a flat surface. A source of torque in the preferred sense of rotation are longitudinal oscillations when the force arm and the reaction force keep an (almost constant) angle generating torque turning the rattleback in the counterclockwise direction.

By simulating equations of motion for small energy initial conditions representing tapping a rattleback on the long side or gently rotating it in the clockwise direction we can see the trajectory of the contact point of the rattleback's body, the force arm and the reaction force generating the counterclockwise torque. The motion of the rattleback is however complex and there is a range of angles close to $90^{\circ}$ and $270^{\circ}$ when transversal oscillations generate a clockwise acting torque responsible for clockwise reversals of the rattleback in agreement with quasi-periodic long-time behaviour of the rattleback starting with low energy IC. There are also 4 directions of tapping IC when the rattleback only oscillates with $\omega_{3} \approx 0$ and has no preferred direction of rotation. Analysis of graphs for the arm of force $\mathbf{a}$, the reaction force $\mathbf{F}_{R}$, angular velocity $\boldsymbol{\omega}$ and for inclination angles $\gamma=\left(\gamma_{1}, \gamma_{2}, \gamma_{3}\right)$ allow to see fine details of motion and how its behaviour is consistent with basic laws of Newtonian mechanics.

\section{Acknowledgement}

M.P. has been supported by grant No. DEC-2013/09/B/ST1/04130 of National Science Centre of Poland. S.R. and M.P. gratefully acknowledge support of the Department of Mathematics of Linköping University for this work and for M.P. visit in Linköping.

\section{References}

[1] I. S. Astapov. On the stability of rotation of the celtic stone. Vestnik Moskov. Univ. Ser. I Mat. Mekh., (2):97-100, 1980.

[2] I. A. Bizyaev, A. V. Borisov, and A. O. Kazakov. Dynamics of the Suslov problem in a gravitational field: reversal and strange attractors. Regul. Chaotic Dyn., 20(5):605-626, 2015.

[3] H. Bondi. The rigid body dynamics of unidirectional spin. Proc. Roy. Soc. London Ser. A, 405(1829):265-274, 1986.

[4] A. V. Borisov, A. Yu. Jalnine, S. P. Kuznetsov, I. R. Sataev, and J. V. Sedova. Dynamical phenomena occurring due to phase volume compression in nonholonomic model of the rattleback. Regul. Chaotic Dyn., 17(6):512-532, 2012.

[5] A. V. Borisov and I. S. Mamaev. Non-holonomic dynamical systems. Integrability, chaos and strange atractors. Institut Komp/yuternykh Issledovaniı̆, Izhevsk, 2002.

[6] Alexey V. Borisov, Alexey O. Kazakov, and Igor R. Sataev. The reversal and chaotic attractor in the nonholonomic model of Chaplygin's top. Regul. Chaotic Dyn., 19(6):718-733, 2014.

[7] A.V. Borisov, A.O. Kazakov, and S.P. Kuznetsov. Nonlinear dynamics of the rattleback: A nonholonomic model. Physics-Uspekhi, 57(5):453-460, 2014.

[8] A.V. Borisov, A.A. Kilin, and I.S. Mamaev. New effects in dynamics of rattlebacks. Doklady Physics, 51(5):272-275, 2006. 
[9] A.V. Borisov and I.S. Mamaev. Strange attractors in rattleback dynamics. Physics-Uspekhi, 46(4):393403, 2003.

[10] W. Case and S. Jalal. The rattleback revisited. Am. J. Phys., 82(7):654-658, 2014.

[11] L. Franti. On the rotational dynamics of the rattleback. Cent. Eur. J. Phys, 11(2):162-172, 2013.

[12] A.S. Gonchenko, S.V. Gonchenko, and A. O. Kazakov. Richness of chaotic dynamics in nonholonomic models of a Celtic stone. Regul. Chaotic Dyn., 18(5):521-538, 2013.

[13] A.V. Karapetian. On the problem of steady motions stability of nonholonomic systems. PMM U.S.S.R, 44(3):295-300, 1980.

[14] V. Kozlov. The phenomenon of reversal in the Euler-Poincaré-Suslov nonholonomic systems. J. Dyn. Control Syst., 22(4):713-724, 2016.

[15] R. E. Lindberg, Jr. and R. W. Longman. On the dynamic behavior of the wobblestone. Acta Mech., 49(1-2):81-94, 1983.

[16] A. P. Markeev. On the dynamics of a solid on an absolutely rough plane. Regul. Chaotic Dyn., 7(2):153-160, 2002.

[17] http://scientific.ics.org.ru/populyarno-o-nauke/sekrety- dinamiki-keltskogo-kamnya, 2015. in Russian.

[18] H. Takano. Spin reversal of a rattleback with viscous friction. Regul. Chaotic Dyn., 19(1):81-99, 2014.

[19] G. T. Walker. On a dynamical top. Quart. J. Pure Appl. Math., 28:175-184, 1896. 